\title{
Política de formação para a prática rural
}

\author{
Aprovado pela Reunião do Conselho Mundial da Wonca
}

9 de junho de 1995

WONCA Working Party on Rural Practice

\section{Resumo}

A escassez mundial de médicos de família rurais contribui diretamente para as dificuldades com prestação de cuidados médicos adequados em áreas rurais e remotas, tanto em países desenvolvidos, quanto nos menos desenvolvidos. A Wonca acredita que há uma necessidade urgente de implementar estratégias para melhorar os serviços de saúde rural em todo o mundo. Isso vai exigir um número suficiente de médicos de família rurais qualificados para fornecer os serviços necessários. Para atingir este objetivo, a Wonca recomenda:

1. Aumentar o número de estudantes de medicina recrutados de áreas rurais.

2. Exposição substancial da prática rural no currículo médico de graduação.

3. Programas de treinamento vocacional em medicina rural específicos, flexíveis, integrados e coordenados.

4. Educação continuada específica adaptada e programas de desenvolvimento profissional que satisfaçam as necessidades identificadas dos médicos de família rurais.

5. Posiçôes acadêmicas apropriadas, desenvolvimento profissional e apoio financeiro para médicos-professores rurais para incentivar a pesquisa e educação rural.

6. Escolas médicas devem assumir a responsabilidade de formar médicos devidamente qualificados para atender às necessidades da sua regiáo geográfica geral, incluindo áreas carentes, e desempenhar um papel fundamental no fornecimento de apoio regional para os profissionais de saúde e cuidados de saúde terciários acessíveis.

7. Desenvolvimento das necessidades rurais adequadas de base e recursos culturalmente sensíveis para cuidados de saúde rural com o envolvimento da comunidade local, com a cooperação regional e apoio do governo.

8. Melhoria das condiçôes profissionais e pessoais/familiares na prática rural para promover a retenção de médicos rurais.

9. Desenvolvimento e implementação de estratégias nacionais de saúde rural, com apoio do governo central. 


\section{Introdução}

Toda prática rural é única e possui seus próprios desafios e recompensas. Uma variedade de definições é usada em todo o mundo, dependendo do contexto local. Na Austrália, a Faculdade de Medicina Rural do (Royal Australian College of General Practitioners) define como prática rural a atividade médica executada fora das zonas urbanas, onde o local de prática obriga alguns médicos generalistas a ter, ou a adquirir, habilidades procedurais e outras, que geralmente não são necessárias na prática urbana.

Continua a haver uma escassez mundial de médicos de família (clínicos gerais) em áreas rurais e remotas, e, em especial, de médicos com as habilidades e os conhecimentos necessários para trabalhar de forma eficaz e confortavelmente nessas áreas. Nos países menos desenvolvidos, a maioria da população está localizada em áreas rurais que podem não ter condições sanitárias básicas, como água potável, alimentação adequada e abrigo, e onde, na melhor das hipóteses, há acesso limitado a serviços modernos de saúde. Os países desenvolvidos também têm significativa escassez de médicos de família rurais, mesmo em países em que há uma oferta global ampla de médicos.

Pessoas que vivem em comunidades rurais e remotas necessitam da segurança de pronto acesso a cuidados médicos em momentos de doença grave ou injúrias. Além disso, médicos e hospitais em comunidades rurais são importantes para a rede econômica e social local. Muitas vezes, o estado de saúde de grupos com necessidades especiais é pior nas zonas rurais que em áreas metropolitanas. Estes incluem os pobres, idosos, mulheres e populaçóes indígenas. Estabelecimento de serviços de medicina de família apoiados por hospitais e outras instalaçóes de saúde fornecem a base para o desenvolvimento de cuidados primários de saúde e programas de promoção da saúde.

Já se sabe que a prestação de serviços médicos por médicos de família amplamente treinados é mais custo-efetiva do que uma gama de profissionais especialistas e outras pessoas que prestem cuidados de saúde primários. Além disso, para os países em desenvolvimento, as melhorias no estado de saúde e o desenvolvimento econômico estão intimamente ligados. Consequentemente, é importante que todas as nações adotem políticas e programas específicos que visem melhorar os serviços de saúde rurais através do aumento do número de médicos de família amplamente qualificados localizados em áreas rurais e remotas.

\section{Vantagens da prática rural}

Médicos rurais identificam uma série de atraçôes-chave na prática rural. Primeiro, a maior variedade da prática que, muitas vezes, inclui obstetrícia, cirurgia, anestesia e medicina de emergência, juntamente com o acesso a hospital e cuidado da doença aguda. Médicos rurais são muito mais propensos a estar cuidando de pacientes individuais, para todos os seus problemas de saúde, de forma contínua; e a cuidar de outros membros da família. Estes cuidados abrangentes e continuados são realidades frequentes no interior.

Para muitos médicos rurais, a segunda grande atração da prática rural é o ambiente rural e o estilo de vida que está associado a uma vida melhor da família, e um bom lugar para criar os filhos, particularmente nos países desenvolvidos. Satisfaçóes sociais da prática rural identificadas por médicos rurais incluem apoio e respeito da comunidade, juntamente com um sentimento de pertencimento a uma comunidade estável, e o prazer da vida ao ar livre, com muitas oportunidades de lazer. Em suma, a prática rural pode oferecer consideráveis recompensas profissionais e satisfaçóes junto com as atraçóes de significativo status social longe das dificuldades da vida na cidade.

\section{Barreiras à entrada na prática rural}

Uma série de barreiras de atitude e perceptivas foi identificada como desencorajadoras à entrada de graduandos em medicina na prática rural. Alguns destes sáo equívocos e outros têm base na realidade. O equívoco fundamental é que a prática rural é, de alguma maneira, uma "prática médica de segunda classe". A maioria dos estudantes de medicina tem um perfil urbano e, assim, náo têm nenhuma experiência pessoal de vida e trabalho rurais. Além disso, a maioria dos professores "sênior" nas escolas médicas tem uma experiência e visão da medicina que os faz enxergar a prática hospitalar de ensino 
como a ideal. Consequentemente, eles assumem que a prática médica em áreas rurais sem as mesmas instalaçóes e apoio, como hospitais de ensino, é de menor padrão.

Um problema importante de atitude é a de "desamparo aprendido". O mais alto que muitos dos novos graduados em medicina aspiram, ao lidar com problemas de saúde, é o de ser capaz de avaliar até para qual especialista encaminhar o paciente. Consequentemente, é uma perspectiva assustadora para eles contemplar a prática rural, na qual eles têm de gerir eles mesmos os problemas, sem acesso imediato às instalaçôes de alta tecnologia médica e a especialistas.

Há uma série de outras barreiras que contribuem para desestimular os novos graduados a almejar a prática rural. Estes incluem a carga de trabalho pesada e longas horas on call ("à disposição" ou "plantão"), que provavelmente continuarão enquanto houver escassez de médicos. A falta de infraestrutura e apoio regional é comum à prática rural, especialmente nos países em desenvolvimento. Além disso, o relativo isolamento profissional, que oferece muitos desafios e recompensas para os médicos rurais, é visto como um fator negativo para muitos estudantes e recém-formados. Muitas vezes, este aspecto é supraenfatizado no contexto de treinamento urbano, em vez do desenvolvimento de conhecimentos e competências individuais e estratégias organizacionais necessárias para atender às necessidades de saúde rural.

Assim como os desestímulos para a prática profissional rural, existem questóes pessoais e familiares também. A prática rural, nomeadamente nas pequenas comunidades, pode ser difícil para o cônjuge do médico. Muitas vezes, o cônjuge é tratado diferentemente de outros membros da comunidade e pode tornar-se pessoalmente isolado. Emprego para o cônjuge e educação para a família, muitas vezes, são problemas significativos na prática rural. Arranjos de liberação local (locum relief) para permitir feriados e educação continuada é, muitas vezes, uma grande dificuldade.

Mesmo para aqueles estudantes e recém-formados que desejam entrar na prática rural, há dificuldades na obtenção de formação adequada e educaçáo permanente. Programas de formação e preparação de graduados para a prática médica rural são relativamente escassos. Uma vez na prática rural, não só a educação continuada é difícil de arranjar, mas, muitas vezes, revela-se de valor limitado para a prática de médicos rurais. Geralmente, os conhecimentos e competências adquiridos através da experiência na prática rural não são devidamente reconhecidos. Isso limita o potencial de desenvolvimento profissional dos médicos que escolhem a prática em áreas rurais.

Juntando todos esses fatores, não é de estranhar que, na opinião de alunos de graduação e de muitos novos graduados em medicina, as vantagens profissionais e sociais da prática rural são suplantadas pelas desvantagens. Para superar esses problemas, deve-se desenvolver uma série de estratégias globais que abordam todas as questóes específicas. Esta política se desenvolve com base na experiência de muitos países ao redor do mundo e forma o quadro de um plano estratégico abrangente para melhorar o recrutamento e retençáo de médicos de família rurais.

\section{Recrutamento e retenção de médicos de família rurais}

O objetivo final deste documento é que haja um número suficiente de médicos qualificados localizados em áreas rurais e remotas para atender às necessidades dos serviços de saúde das pessoas a quem servem. Embora o foco principal do documento seja a educaçáo e a formação para a prática rural, isto deve ser visto no contexto mais amplo de recrutamento e retenção de médicos na prática rural. Há uma necessidade de estabelecer uma via integrada de carreira de educação e formação para a prática rural, iniciando no nível de pré-graduação, continuando com a educação médica através de cursos de formação profissional com treinamento vocacional específico para a prática rural, seguido por educação continuada e universitária adequadas, estruturas para a prática e suporte familiar.

Em última análise, o recrutamento para a prática rural só aumentará quando os estudantes e médicos recém-formados enxergarem a prática rural como uma opção positiva de carreira. As séries de estratégias descritas neste documento pretendem que isso aconteça sensibilizando precocemente alunos para a medicina rural e oferecendo ensino clínico apropriado na última parte do curso de graduação e no período imediato após a graduação.

Retenção na prática rural pode ser melhorada através de formação continuada específica e programas de desenvolvimento profissional e a oportunidade de prosseguir o ensino superior universitário enquanto permanece na prática rural.

Além de questões de educação e treinamento, há uma série de outros fatores que requerem atenção em qualquer programa para melhorar o recrutamento e retenção para a prática rural. Condiçóes de trabalho razoáveis, incluindo um equilíbrio entre a carga de trabalho, de plantão e tempo livre, são essenciais. Cobertura profissional transversal para férias ou lazer 
confiável ou locum relief é uma questáo fundamental. Também é preciso que haja recompensa financeira apropriada para a complexidade dos serviços prestados e o grau de responsabilidade clínica assumida pelo médico. Outros aspectos financeiros incluem os custos adicionais de viver em comunidades rurais com a necessidade de transporte para grandes centros para educação continuada e desenvolvimento profissional. Proporcionar uma boa educação para os filhos do médico pode ser difícil e caro.

Além disso, a retenção de médicos rurais depende muito da satisfação do cônjuge e familiares dos médicos. Muitas vezes, as razôes para os médicos rurais retornarem para a cidade se relacionam com preocupaçóes com o cônjuge e familiares. Consequentemente, a estes é dada atençáo específica neste documento.

\section{Educação na graduação}

Experiências em todo o mundo mostram que os estudantes de origem rural são muito mais propensos a se inserir na prática rural após a graduação. $\mathrm{Na}$ maioria dos cursos de medicina atuais, a proporção de estudantes de origem rural é significativamente menor do que a proporção da população que vive no país. Claramente, uma estratégia importante para aumentar o número de médicos rurais envolve o recrutamento de mais estudantes de medicina advindos do âmbito rural.

Para que isso ocorra, alunos do ensino médio de áreas rurais precisam ser encorajados a considerar a medicina como uma opçáo de carreira e se candidatarem a entrar nos cursos de medicina. Consequentemente, há a necessidade de programas específicos que promovam a medicina nas escolas secundárias rurais. Em muitas áreas rurais, o padrão acadêmico das escolas secundárias pode não ser suficientemente alto para que os seus diplomados possam se qualificar para o ingresso nas escolas médicas. De modo que programas que identifiquem potenciais estudantes de medicina e possam auxiliá-los com o ensino secundário, em preparação para a entrada na escola médica, precisam ser desenvolvidos.

A fim de assegurar que uma proporção adequada dos estudantes de origem rural seja recrutada em escolas de medicina, é necessário que haja mecanismos específicos incluídos no processo de seleção. Critérios para a seleção com base em metas além de outros critérios estão evoluindo. Processos de seleção que incluem entrevistas de candidatos, nas quais é oferecido reconhecimento e crédito para aqueles provindos de ambientes rurais, devem ser incentivados. Metas específicas para a admissão de alunos de origem rural podem ser necessárias.

Depois de possuir origem rural, o fator mais forte associado com o ingresso na prática rural é possuir experiência clínica de graduação e de pós-graduação em um cenário rural. Consequentemente, a exposição rural para todos os alunos de graduação médica deve ser maximizada. A exposição positiva precoce à prática rural irá incentivar mais estudantes a desenvolver interesse na medicina rural como uma opçáo de carreira e promover melhor compreensão da prática rural para os outros. Todos os alunos devem ser introduzidos às questóes de saúde rural no início do curso médico e participar de rodízios clínicos em hospitais rurais e em clínicas de prática rural familiar mais adiante no curso.

Como os praticantes da medicina rural fornecem uma ampla oferta de serviços, com maior variedade do que seus colegas metropolitanos, estágios de prática rural proporcionam aos alunos a oportunidade de desenvolver uma gama de habilidades clínicas. Estes incluem habilidades procedurais diagnósticas e terapêuticas, bem como habilidades de julgamento clínico e autossuficiência no cenário prático. Esta experiência rural também ajuda os alunos a identificar as suas próprias necessidades de aprendizagem.

Além disso, os alunos devem ser incentivados a realizar estágios opcionais e eletivos em saúde rural, que vão desde práticas em hospitais rurais, medicina de família rural e outros serviços de saúde rural.

"Clubes de Prática Rural" encorajam estudantes de origem urbana a desenvolver o interesse pela prática rural e apoiar os alunos de origem rural a se ajustar aos desafios da vida na cidade e aos estudos universitários. Estudantes de origem rural deveriam ser assistidos ainda mais através de esquemas com médicos rurais como mentor: cada um seria anexado a um médico que pratica medicina na zona rural ou na regiáo da qual o aluno provém.

O mentor fornece ao estudante o apoio pessoal e incentivo, bem como um modelo profissional.

Para os alunos que demonstram um comprometimento precoce com a prática rural, entâo, um "direcionamento para a medicina rural" na escola médica é recomendado. Isso pode assumir a forma de um a três anos, do currículo médico completo, realizados no meio rural ou uma sequência de complementos rurais interligados através dos componentes clínicos do currículo. 
Escolas médicas descentralizadas que permitam ao estudante de medicina realizar a maior parte ou todo o seu estudo em centros localizados fora das principais áreas metropolitanas são mais susceptíveis de atrair estudantes de áreas rurais e de serem bem sucedidas na produção de médicos para a prática nas áreas rurais.

$\mathrm{Na}$ educação médica, um currículo de medicina de família baseado na comunidade deve ser encorajado e deve incluir de maneira significativa o conteúdo rural. As escolas de medicina deveriam assumir a responsabilidade de educar os médicos devidamente treinados para atender às necessidades da sua regiáo geográfica geral, incluindo áreas carentes. Do mesmo modo que devem desempenhar um papel fundamental no fornecimento de apoio regional para os profissionais de saúde e na provisão de cuidados de saúde em nível terciário. A inclusão de médicos rurais como educadores e pesquisadores é essencial para o desenvolvimento de melhor compreensão e uma atitude de apoio para a prática rural.

O desenvolvimento do ensino de graduação e pós-graduação no treinamento para a prática rural é muito facilitada pela criação de Centros de Educação Médica Rural. Estes centros devem ser estabelecidos em áreas rurais com o objetivo de coordenar o ensino de graduação, a formação profissional, a educação continuada e os estudos universitários de pós-graduação para os médicos rurais. Uma função importante desses centros é facilitar o desenvolvimento de relaçôes recíprocas entre os hospitais/clínicas rurais e escolas médicas/hospitais de ensino. A criação destes centros oferece a oportunidade para os médicos de família rurais serem ativamente envolvidos no ensino dos alunos e no treinamento do exercício de ensino profissional. Eles também fornecem um foco para outros desenvolvimentos acadêmicos, incluindo a pesquisa em saúde rural.

\section{Formação profissional após a graduação}

Médicos de família rurais geralmente proporcionam uma gama de serviços mais ampla do que os seus pares metropolitanos. Consequentemente, há uma necessidade de programas de residência com formação específica para a prática rural que preparem novos graduados em medicina para uma carreira no meio rural.

Sempre que possível, a formação para a prática rural deve ocorrer no meio rural com base em hospitais regionais rurais e medicina de família rural. Adicionalmente ao treinamento padrão para a medicina de família, a formação prática profissional rural requer especial destaque para: aprendizagem prática (hands-on) de habilidades procedurais o espectro de doenças em comunidades rurais e remotas; a sociologia e a psicologia das comunidades rurais e remotas; e aspectos profissionais e pessoais da vida e do trabalho em pequenas comunidades rurais.

Vagas de treinamento para habilidades avançadas de prática rural em medicina de emergência, anestesia, cirurgia, obstetrícia processual e outros precisam ser desenvolvidas e adequadamente financiadas. Dependendo da intensidade do programa, essa formação pode envolver de um a dois anos de tempo de treinamento adicional para além de treinamento básico de medicina de família.

Deve-se considerar o reconhecimento à formação profissional rural na forma de certificaçáo em medicina rural. A oportunidade de realizar algum treinamento em outros países pode ampliar a experiência e ajudar a desenvolver novas abordagens para a prática médica, a educação médica e a prestação de cuidados de saúde.

\section{Educação permanente e apoio profissional}

A maioria dos praticantes rurais experimentam grandes dificuldades em conseguir facilidades locais para participar de atividades de educação permanente. Muitas vezes, os médicos de família rurais, quando se inserem em programas de educação continuada, os acham de pouco valor para eles, pois não se encontram em nível adequado.

Existe uma necessidade de que os programas de educação continuada e desenvolvimento profissional sejam especificamente adaptados para atender às necessidades dos médicos de família das zonas rurais. Estes programas devem ser desenvolvidos por médicos de zonas rurais para médicos rurais. Centros de Educação Médica Rural fornecem um foco muito apropriado para o desenvolvimento de tais programas de educação continuada.

Estes programas devem reconhecer os conhecimentos pré-existentes e as competências dos médicos de família rurais, que, muitas vezes, são desenvolvidos através do manejo de problemas clínicos no isolamento profissional relativo e não através de 
treinamentos formais. Os programas devem ser sensíveis às necessidades específicas de aprendizagem dos médicos, que geralmente envolvem um foco que é prático, baseadas em casos, e orientadas por problemas. O objetivo de tais programas de educação continuada deve ser o de capacitar o aluno e, portanto, estender e expandir os conhecimentos médicos e habilidades clínicas.

Os programas de educação continuada também devem ser acessíveis aos médicos rurais, o que significa localizá-los em centros regionais da zona rural ao invés de grandes cidades. Além disso, o uso de métodos de educação a distância para levar a educação continuada para os profissionais rurais deve ser incentivado. Isso inclui náo apenas os tradicionais materiais publicados, mas também o uso de novas tecnologias, incluindo teleconferência, correios eletrônicos, televisão por satélite, e outros desenvolvimentos em tecnologia da informaçáo moderna.

Outra importante forma de educação continuada e desenvolvimento profissional são rodízios clínicos "hand-on" de curto prazo em hospitais maiores. Estes devem ser incentivados e facilitados por meio da vinculação com os especialistas nesses hospitais. A liberação do trabalho talvez possa ser facilitada por esquemas locais de rotação de liberaçôes, nos quais um grupo de médicos rurais compartilha um local de estágio.

A oportunidade de ter licença remunerada e realizar intercâmbios em outros países pode ampliar a experiência prática de médicos rurais e ajudar a desenvolver novas abordagens para a prática médica, a educação médica e para a prestação de cuidados de saúde.

\section{Estudos superiores da universidade}

Atualmente não há sentido de progressão na carreira para os médicos que seguem a prática rural, e àqueles que mais tarde desejam seguir uma carreira acadêmica são dados poucos créditos para o conhecimento e experiência adquiridos durante a prática no interior do país. Há necessidade de desenvolver diplomas universitários apropriados de pós-graduação e graduação que forneçam um meio para a progressáo da carreira em pesquisa, educação ou administraçáo.

Além disso, tais programas de pós-graduação vão ajudar na criação de uma classe de profissionais rurais academicamente treinados para serem o corpo clínico dos Centros de Educação Médica Rurais e de outras unidades acadêmicas de saúde rural.

\section{Apoio financeiro e material}

Como mencionado anteriormente, a prática em áreas remotas e rurais tem muitas desvantagens financeiras. A fim de recrutar e reter os médicos em ambientes de prática remotos e rurais, estas questóes financeiras precisam ser abordadas. Isto pode assumir a forma de pagamento adicional, reconhecendo o maior nível de responsabilidade clínica e serviços prestados; pagamentos específicos de incentivos para a prática em áreas carentes; assistência financeira com a educação, alojamento e viagem para o médico e sua família, e assim em diante.

Outra forma de apoio material é a disponibilização de instalaçôes e equipamentos para a prática médica. Muitas comunidades rurais fornecem tais facilidades para auxiliar na atração de médicos.

É mais provável que um médico se mantenha a longo prazo em um ambiente de prática rural no qual ele ou ela não é o único fornecedor de serviços médicos. Consequentemente, dois ou três grupos médicos devem ser incentivados sempre que necessário através do apoio financeiro direto, de modo a sustentar a viabilidade econômica da prática. A fim de fornecer cuidados de saúde primária eficaz, os médicos rurais requerem a assistência de enfermeiros devidamente formados e de outros profissionais de saúde, combinando facilidades para os médicos e outros profissionais em centros comunitários de saúde rural que promovam a prestação de cuidados integrados de saúde.

Depois de um médico, a próxima prioridade de serviço de saúde para uma comunidade rural é um hospital que ofereça cuidados médicos para situaçóes agudas, cirúrgicas, obstétricas e cuidados pediátricos. Muitos desses hospitais foram construídos e equipados com um apoio financeiro considerável da comunidade local. O hospital é importante também para a economia da cidade como um grande empregador e comprador de bens e serviços dentro da comunidade. Médicos das zonas rurais necessitam de facilidades e privilégios para fornecer os serviços necessários para os quais são treinados e 
competentes. Grandes dificuldades em comunidades rurais podem resultar da imposiçấo das autoridades reguladoras centrais pela certificação excessiva ou pela exigência de especialização para a execução de procedimentos.

A prestação geral de serviços de saúde pode ser aprimorada pela integração entre os médicos e pelo compartilhamento de facilidades de cuidados de saúde e profissionais entre diversas comunidades. Há também a responsabilidade governamental de garantir que o sistema de saúde forneça instalaçôes físicas adequadas e serviços para atender às necessidades das comunidades rurais e remotas.

\section{Família e suporte conjugal}

Para o médico da família rural, há um grande desafio em ser o conselheiro confidencial médico no consultório e amigo do ambiente social e de lazer na comunidade. Para os cônjuges dos médicos, isto pode ser ainda mais difícil, pois os membros da comunidade tendem a tratá-los de forma diferente, devido a sua conexão com o médico. Em muitos aspectos, o cônjuge do praticante rural pode ser socialmente mais isolado do que o médico. Consequentemente, há uma necessidade de estratégias específicas para fornecer apoio pessoal para os cônjuges dos médicos. Também os cônjuges têm, muitas vezes, dificuldades na obtenção de emprego e/ou realização de objetivos de carreira. Estratégias para atender a essas necessidades devem ser incluídas.

Para a família do médico, há dificuldades com a educação e emprego posteriores. Estratégias auxiliares de apoio educacional e de financiamento para ir longe buscar a educação deve ser incluído em programas de apoio às famílias dos médicos.

Os períodos de plantâo com chamadas frequentes levam à interrupção dos espaços e momentos familiares tão grandes, que há necessidade de mais tempo do que os períodos habituais de férias e recreação para os médicos rurais e suas famílias. Programas de assistência devem incluir o local apropriado para o repouso e assistência financeira para permitir a recreaçáa fora da comunidade rural.

\section{Apoio nacional}

O apoio do governo central é essencial para a prestação de cuidados de saúde acessíveis, particularmente em áreas rurais carentes. Os governos nacionais precisam desenvolver e implantar estratégias nacionais de saúde rural. Isto requer a cooperação das comunidades, médicos e outros profissionais de saúde, hospitais, escolas médicas, organizaçóes profissionais, e os governos. Cuidados de saúde rurais deveriam ser dotados de recursos e mecanismos de financiamento deveriam ser desenvolvidos para satisfazer às necessidades das populaçóes rurais. A criação de Organizaçóes de Pesquisa Nacional de Saúde Rural pode facilitar este processo.

\section{Conclusão e recomendações}

A Wonca acredita que há uma necessidade urgente de implantar estratégias para melhorar os serviços de saúde rural em todo o mundo. Para alcançar isso, é necessário um número suficiente de médicos de família rurais qualificados para fornecer os serviços médicos necessários. Este documento traçou uma série de questôes fundamentais de interesse em relação à formação para a prática rural.

Verificou-se que a produção de mais e mais médicos não leva a um fluxo de médicos das cidades para o meio rural. A fim de aumentar o número e a qualidade de médicos rurais, é necessário implantar uma série de estratégias destinadas a estabelecer uma via integrada de carreira de educação e formação para a prática rural. A longo prazo, é a única abordagem estratégica susceptível de melhorar o recrutamento e a retenção de médicos de família rurais. Para atingir este objetivo, a Wonca recomenda: 
1. Aumentar o número de estudantes de medicina recrutados a partir das áreas rurais. Estratégias podem incluir:

1.1. Introdução de programas de promoção da medicina como uma carreira para alunos do ensino médio rural.

1.2. Criação de bolsas de estudo e programas de apoio educativo que identifiquem potenciais estudantes de medicina nas áreas rurais e ajudem-nos com educação secundária e terciária, em preparação para a entrada na escola médica.

1.3. Processos de seleção que incentivem a admissão de estudantes das áreas rurais.

1.3.1. Processos de seleção, incluindo entrevistas, devem dar reconhecimento específico e crédito para origem, experiência e interesse rurais.

1.3.2. Metas específicas para os estudantes de origem rural podem ser necessárias.

\section{Exposiçáo substancial à prática rural no currículo de graduaçáo médica. Isto pode ser conseguido através de:}

2.1. Estabelecimento de "Clubes de Prática Rural" que incentivam alunos de origem urbana a desenvolver interesse na prática rural e apoio aos estudantes de origem rural para responder aos desafios da vida urbana e dos estudos universitários.

2.2. Esquemas de tutoria médica rural que ofereça aos estudantes de origem rural apoio pessoal e incentivo, por um médico de família rural designado.

2.3. Introdução precoce à questôes de saúde rural no currículo, incluindo anexos específicos de medicina rural prática para os alunos no início do curso de medicina.

2.4. Rodízios clínicos em internatos para hospitais rurais e prática familiar rural no final do curso.

2.5. Um eixo de medicina rural para um grupo selecionado de estudantes que demonstra envolvimento precoce com a prática rural. Isso pode assumir a forma de:

2.5.1. De um a três anos de currículo médico completo, realizados no meio rural.

2.5.2. Um segmento de estágios rurais eletivos interligados através dos componentes clínicos do currículo.

2.6. Descentralizar escolas médicas que permitem que os alunos façam a maior parte ou toda a sua educação escolar em centros médicos fora das grandes áreas metropolitanas.

3. Programas de treinamento vocacional para prática rural específicos, flexíveis, integrados e coordenados. Estes programas devem:

3.1. Ser direcionados por necessidades, baseados em evidências, e aluno-centrados.

3.2. Ter um corpo docente adequado, hospital, e apoio financeiro.

3.3. Dar ênfase particular à formação em competências procedurais e a um currículo adequado à prática rural, além de uma sólida base de medicina de família.

3.4. Oferecer uma porção importante da formação dentro do contexto rural.

3.5. Fornecer oportunidade e financiamento para o treinamento de competências rurais avançadas em emergência, anestesia, cirurgia, obstetrícia prática e outros.

3.6. Oferecer oportunidades para residentes regulares de medicina de família experimentar as alegrias e desafios da prática da família rural.

4. Educaçáo contínua específica adaptada e programas de desenvolvimento profissional que respondam às necessidades identificadas dos médicos de família rurais.

4.1. Programas de educação médica continuada devem ser acessíveis para os profissionais rurais pela locação em centros regionais rurais e, onde apropriado, fazendo uso de métodos de educação a distância, incluindo tecnologia da informação moderna.

4.2. Geralmente, programas de educação médica continuada devem ser desenvolvidos por médicos rurais para médicos rurais.

4.3. Desenvolvimento de graduação e diplomas de pós-graduação universitários apropriados, disponíveis através de educação a distância, de modo a permitir a médicos rurais mais isolados o prosseguimento de estudos universitários superiores sem sair de suas cidades ou práticas. 
5. Posiçóes acadêmicas apropriadas, o desenvolvimento profissional e apoio financeiro para médicos-professores rurais incentivarem a investigaçáo em saúde e educaçáo rural.

5.1. Educação Médica Rural e Centros de Pesquisa devem ser estabelecidos em áreas rurais com o objetivo de coordenar o ensino de graduação, a formação profissional de pós-graduação e a educação médica continuada para os médicos rurais. Tais centros facilitam grandemente a implementação de todas as recomendaçóes anteriores. Uma importante consequência do estabelecimento de Educação Médica Rural e Centros de Investigação é o desenvolvimento de relaçóes recíprocas entre os hospitais e práticas rurais e escolas médicas/hospitais de ensino.

6. Escolas médicas devem assumir a responsabilidade de educar médicos devidamente qualificados para atender às necessidades da sua regiáo geográfica em geral, incluindo áreas carentes, e deve desempenhar um papel fundamental no provimento de apoio regional para profissionais de saúde e nível terciário de saúde acessível.

7. Desenvolvimento de recursos para a saúde rural baseados em necessidades e culturalmente sensíveis, com o envolvimento da comunidade local, cooperaçáo regional e apoio do governo.

7.1. Fornecer financiamento adequado para desenvolver e manter hospitais e outros serviços de saúde e serviços de referência para atender às necessidades das pessoas em comunidades rurais e remotas.

7.2. Criar centros de saúde comunitários rurais, com instalaçôes e apoio para os médicos e outros profissionais de saúde.

8. Melhoria das condiçóes profissionais e pessoais/familiares na prática rural para promover a retençáo de médicos rurais. Estratégias incluem:

8.1. Esquemas de liberaçáo local devem ser estabelecidos para permitir a liberaçáo dos médicos de família rurais para realizar a educação continuada, bem como recreação e outras formas de viagem.

8.2. Apoio financeiro orientado para a prática rural, tais como:

8.2.1. Modelos de financiamento que ofereçam segurança e flexibilidade para o médico e reconheçam o médico como um recurso da comunidade.

8.2.2. Os pagamentos complementares para os profissionais rurais em reconhecimento ao alto nível de responsabilidade clínica, serviços prestados e nas chamadas sob demanda.

8.2.3. Pagamentos de incentivos específicos para a prática em áreas isoladas/carentes.

8.2.4. O apoio financeiro para manter a viabilidade econômica de pelo menos dois médicos trabalhando juntos em uma zona rural.

8.2.5. O financiamento para viagens e outros custos para o médico para submeter-se à educação médica continuada.

8.3. Programas específicos para atender às necessidades dos cônjuges dos médicos rurais e famílias, tais como:

8.3.1. Redes de apoio familiar e ao cônjuge.

8.3.2. Assistência financeira com acomodação para o médico e familiares.

8.3.3. Assistência financeira para facilitar a educação da família do médico.

8.3.4. Financiamento para permitir viagens do médico e da família para recreação e outras formas de viagem e para visitar familiares em processo de ensino secundário ou terciário.

8.3.5. Assistência no desenvolvimento de oportunidades de emprego para o cônjuge do médico.

9. Desenvolvimento e implantaçáo de estratégias nacionais de saúde rural, com o apoio do governo central. Isto requer:

9.1. Envolvimento cooperativo das comunidades, médicos e outros profissionais de saúde, hospitais, escolas médicas, organizaçóes profissionais e os governos, em todos os níveis. O estabelecimento de organizações nacionais de pesquisa e educação em saúde rural pode facilitar este processo. 


\section{Referências}

Este documento da Wonca é baseado em experiências em muitos países ao redor do mundo. A seguinte lista de referências destaca questóes fundamentais:

1. Australian Health Ministers Conference. National Rural Health Strategy. Canberra: Australian Government Publishing Service; 1994.

2. Rural Undergraduate Steering Committee. Rural Doctors: Reforming undergraduate medical education for rural practice. Australian Commonwealth Department of Human Services and Health. Canberra: Australian Government Publishing Service; 1994.

3. Rural medicine design project. Training curriculum surgery, anaesthesia and obstetrics for rural general practice. Sydney: Faculty of Rural Medicine, Royal Australian College of General Practitioners; 1992.

4. Association of American Medical Colleges. Rural Health : A challenge for medical education. Proceedings of 1990 invitational symposium. Acad. Med. 1990; 65(Suppl.): 1-126. PMid:2294917. http://dx.doi.org/10.1097/00001888-199012000-00024

5. Littlemeyer M, Martin D. Academic Initiatives to address physician supply in rural areas in the United States : A compendium. Washington: Association of American Medical Colleges; 1991.

6. American Academy of Family Physicians. Rural Family Practice: You can make a difference. Kansas City: American Academy of Family Physicians; 1989.

7. Canadian Medical Association. Report of the advisory panel on the provision of medical services in underserviced regions. Ottawa: Canadian Medical Association; 1992.

8. Blackwood R, McNab J. A portrait of rural family practice: Problems and Priorities. Toronto: College of Family Physicians of Canada; 1991. PMCid:1298622.

9. Stiratanaban A, Sangprasert B. The Rural Area Project (RAP) in Thailand: curriculum development. Med. Educ. 1983; 17: 374-377. http://dx.doi. org/10.1111/j.1365-2923.1983.tb01123.x

10. Carter RG. The relation between personal characteristics of physicians and practice location in Manitoba. CMAJ. 1987; 136: 366-368. PMid:3815198 PMCid:1492066.

11. Asuzu MC. The influence of undergraduate clinical training on the attitude of medical students to rural medical practice in Nigeria. Afr. J. Med. \& Med. Sci. 1989; 18: 245-250. PMid:2558554.

12. Poulose KP and Natarajan PK. Re-orientation of medical education in India past, present and future. Indian J. Pub. Health. 1989; 33 : 55-58. PMid:2641747.

13. Hickner JM. Training for rural practice in Australia 1990. Med. J. Australia. 1991; 154: 111-118. PMid:1986187.

14. Rosenblatt RA, Whitcomb ME, Cullen TJ, Lishner DM and Hart LG. Which medical schools produce rural physicians? JAMA. 1992; 268: $1559-1565$. PMid:1308662. http://dx.doi.org/10.1001/jama.1992.03490120073031

15. Strasser RP. Attitudes of Victorian rural general practitioners to country practice and training. Aust. Fam. Phys.. 1992; 21(7): 808-812.

16. Umland B, Waterman R, Wiese W, Duban S, Mennin S, Kaufman A. Learning from a rural physician program in China. Acad. Med. 1992; 67: 307-309. PMid:1575861. http://dx.doi.org/10.1097/00001888-199205000-00004

17. Magnus JH and Tollan A. Rural doctor recruitment: does medical education in rural districts recruit doctors to rural areas? Med. Educ. 1993; $25: 250-253$. http://dx.doi.org/10.1111/j.1365-2923.1993.tb00264.x

18. Gray JD, Steeves LC and Blackburn JW. The Dalhousie University experience of training residents in many small communities. Acad. Med. 1994; 69(10): 847-851. http://dx.doi.org/10.1097/00001888-199410000-00016

Uma lista extensa de artigos publicados sobre a educação para a prática rural foram recuperados, confrontados e anotados pelo Dr. James Rourke. Esta publicação "Educação para a prática rural: Metas e oportunidades: uma bibliografia anotada" está disponível sob pagamento através do Australian Rural Health Research Institute Moe, Victoria 3825 Austrália

Tradução para a versão brasileira: Leonardo Cançado Monteiro Savassi e Magda Moura de Almeida. Membros do Grupo de Trabalho em Medicina Rural da Sociedade Brasileira de Medicina de Família e Comunidade.

Rio de Janeiro: SBMFC, 2011. 